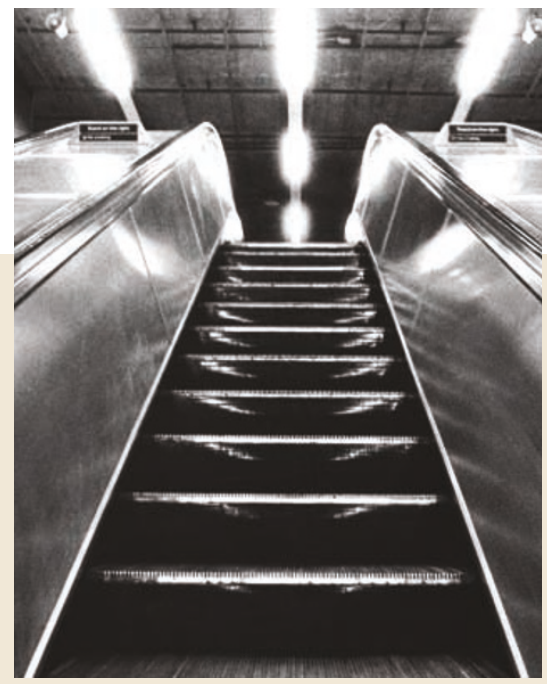

Quality control of newly synthesized membrane proteins, such as G-proteincoupled receptors (GPCRs), in the endoplasmic reticulum (ER) is important in ensuring that only properly folded proteins reach the cell surface. Even subtle mutations that would not markedly affect protein function can lead to ER retention and subsequent degradation of the mutant protein, which can have adverse results; for example, nephrogenic diabetes insipidus (NDI) is caused by such mutations in the $\mathrm{V} 2$ vasopressin receptor (V2R). Moreover, it also seems that a proportion of newly synthesized non-mutant proteins might never attain their correct structure owing to inefficient processing and therefore be degraded. As Petäjä-Repo, Bouvier and colleagues describe in The EMBO Journal, membrane-permeable GPCR ligands can facilitate maturation and ER export of GPCRs, a phenomenon that could have significant implications for drugs that target GPCRs and other membrane proteins.

Prompted by previous experiments that had shown that membrane-permeable antagonists of the V2R could functionally rescue several receptor mutants that were normally retained in the ER, the authors studied the effects of various opioid ligands on the maturation and ER export of the $\delta$ opioid receptor, which is known to be inefficiently processed.

Naltrexone - a membrane-permeable antagonist - doubled the amount of mature receptors, an effect that was shown not to be due to an influence on protein synthesis or on stabilization of proteins at the cell surface.

Other membrane-permeable opioid antagonists and agonists also favoured maturation of the $\delta$ opioid receptor, but a membrane-permeable antagonist of the unrelated V2R did not, indicating that receptor occupancy is required to influence receptor maturation. The authors propose that binding of receptor ligands might promote the correct folding of newly synthesized receptors, decreasing the possibility of degradation and ultimately leading to an increase in the steady-state level of functional receptors at the cell surface. So, these 'pharmacological chaperones' might not just be potentially useful in diseases caused by ER retention of mutant proteins, such as NDI, but also represent a novel mechanism by which receptor agonists and antagonists might mediate changes in receptor density and cell responsiveness to drugs.

Peter Kirkpatrick

(iv) References and links ORIGINAL RESEARCH PAPER Petäjä-Repo, U. E. et al. Ligands act as pharmacological chaperones and increase the efficiency of $\delta$ opioid receptor maturation. EMBO J. 21, 1628-1637 (2002)

FURTHER READING Morello, J.-P. et al. Pharmacological chaperones rescue cell-surface expression and function of misfolded V2 vasopressin receptor mutants.

J. Clin. Invest. 105, 887-895 (2000)

\section{MICROBIAL DISEASES}

\section{Squatters' rights need ironing out}

When bacteria invade tissues, they can attach and colonize mucosal surfaces, such as sinuses and lungs, resulting in the formation of biofilms, which are specialized, matrix-encased communities that are usually resistant to killing

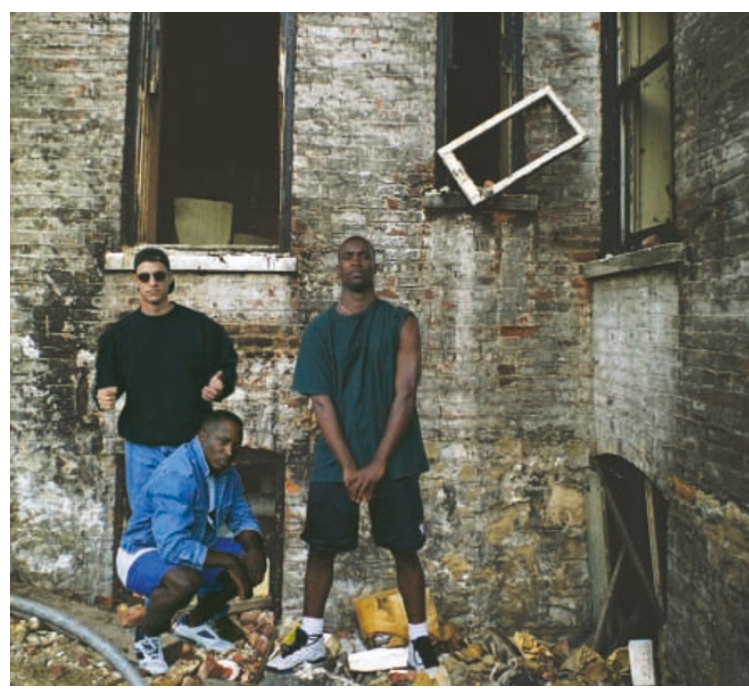

by both the host-defence system and antibiotics. Research in Nature shows that bacteria require iron to transform from mobile 'ramblers' or ' flyers' to groups of stationary, biofilm-forming 'squatters'. The iron chelator lactoferrin, which is an abundant constituent of human secretions, forms part of the normal host-defence mechanisms against biofilm formation by preventing bacterial squatting.

Despite continual exposure to pathogenic bacteria, healthy mucosal surfaces resist biofilm infections. However, in many chronic infections, bacteria live in biofilms, and they are a major medical problem. Airway infection by Pseudomonas aeruginosa in patients with cystic fibrosis is a prime example of a biofilm infection. Once this infection develops, the bacteria colonize the airways for life, causing lung destruction and eventual death. Lactoferrin is known to limit bacterial growth by sequestering iron, but the authors examined whether subinhibitory concentrations of lactoferrin, which did not affect the growth rate of $P$. aeruginosa, had any effect on biofilm formation.

In a continuous culture-flow device, the presence of lactoferrin, or iron-saturated lactoferrin, prevented the development of $P$. aeruginosa biofilms, although lactoferrin could not alter their structure once they were formed. The iron chelator deferoxamine gave similar results, which indicates that lactoferrin might block biofilm formation in $P$. aeruginosa by sequestering free iron.
Lactoferrin markedly altered bacterial movement. Three types of motility were defined: bacteria that moved away from their division site were known as ramblers; bacteria that were swept away by the flow of medium were called flyers; and those that remained stationary from the time that they were created to the time that they divided were known as squatters. In the absence of lactoferrin, the predominant behaviour was squatting, whereas in the presence of lactoferrin, rambling was dominant. These behaviours became more prevalent in subsequent generations. Iron chelation stimulates increased twitching - a specialized form of surface motility. In twitching motility assays, as free-iron levels decreased, twitching motility increased. Furthermore, in a $P$. aeruginosa mutant that could not twitch, biofilms were formed in both the presence and absence of lactoferrin. This confirmed that lactoferrin prevented biofilm formation by stimulating twitching motility.

This work gives new insight into host defence and the formation of biofilms. Whereas iron chelation could be helpful for preventing the growth of new biofilms, it is unlikely to have therapeutic applications for patients with established biofilm infections.

(2) References and links Melanie Brazil ORIGINAL RESEARCH PAPER Singh, P. K. et al. A component of innate immunity prevents bacterial biofilm development. Nature 417, 552-555 (2002) development. Nature 417, $552-555$ (2002)
WEB SITES Encyclopedia of Life Sciences: http///wmw.els net Biofilms 